\title{
Trade-offs in the production and end-use of biochar and bio- oil from the solid waste generated from the olive oil industry in Australia
}

\author{
A. El Hanandeh \\ Griffith School of Engineering, Griffith University, Nathan QLD 4111, Australia \\ (a.elhanandeh@griffith.edu.au)
}

\begin{abstract}
The olive oil industry in Australia has been growing at a rapid rate over the past decade. This growth brings along with it new challenges. The solid waste, produced from the olive oil mills, is particularly difficult to manage. Pyrolysis is a process which may be employed to generate biochar, bio-oil and gas from biomass. The proportions and in fact the quality of the different products depend on the process parameters such as final temperature, heating rate, particle size and pyrolysis method. The change in the products quantities and qualities affect the potential revenue from the sale of these products as well as the potential environmental credits due to the altered use and or product properties. This paper investigates some of the economic and environmental trade-offs in the production and end-use of the biochar and bio-oil from the olive-oil solid waste in Australia. Special attention is paid to the impact of policies such as renewable energy targets, carbon tax and carbon sequestration credits on agricultural lands. Under the current government policies, the optimal utilisation of the pyrolysis products is limited due to the lack of incentives for the use of bio-char as a soil amendment on agricultural soils.
\end{abstract}

Keywords: pyrolysis; olive husk; bio-oil; biochar; carbon sequestration; carbon tax 


\section{INTRODUCTION}

\subsection{Olive Oil Industry in Australia}

The olive oil industry in Australia has witnessed a rapid growth over the past decade driven by stronger demand in the local Australian market and a rapid growth in demand in the Asian region. Figure 1 shows the growth in the Australian olive oil production and export.

In 2009 season, Victoria accounted for nearly half of the olive oil production in Australia followed by Western Australia (24\%) and South Australia (14.5\%). Nevertheless, olive groves are grown in all temperate agricultural regions of Australia.

\subsection{Olive Oil Production and Solid Waste Generation}

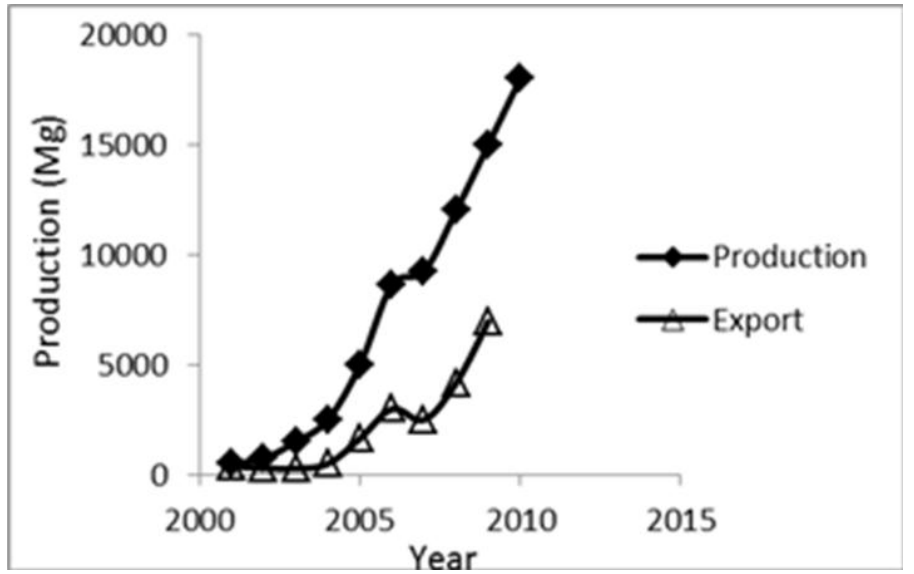

Figure 1. Olive oil production and export in Australia (Data source: Australian Olive Association Ltd).

Olive oil production is associated with significant environmental impacts. Besides olive oil, the process leaves behind highly polluting wastewater and solid waste. The quantities and characteristics of the waste stream depend on the processing method. The modern continuous 2-phase and 3-phase olive oil mills are used by the young olive oil industry with the 2-phase being more popular. Table 1 presents a summary of the olive oil processing products from the two common methods in Australia.

The solid waste generated from olive oil mills is characterised by its phytotoxicity, hydrophobicity, salinity and acidity. The physical characteristics of the solid waste from the 2-phase and 3-phase mills in Australia differ markedly. 2-phase solid waste has a thick paste consistency and dries to a hard solid with low porosity and small particle size. On the other hand, 3-phase husk has larger particle size and dries to a loose solid with relatively higher porosity (Nair and Markham 2008). This is generally because the 2-phase olive husk has a higher moisture and higher sugar content which makes it stickier and harder to dry (Arjona, Garcia et al. 1999). Based on the 2010 olive oil production figures, it is estimated that more than 70 thousand $\mathrm{Mg}_{\text {wet }}$ olive husk was generated Australia wide. Currently, olive husk is underutilised and is considered a liability as it costs farmers around \$15 per Mg to dispose of it to landfills (Dally and Mullinger 2002). Other methods of disposal such as spreading raw olive husk over agricultural land is highly discouraged due to its adverse effects on the agricultural soil such as increasing the soil hydrophobicity, increasing the C:N ratio and acidity (Roig, Cayuela et al. 2006; Kavdir and Killi 2008).

\subsection{Pyrolysis of Olive Husk}

Pyrolysis can be defined as the direct thermal decomposition of organic material in the absence of oxygen to produce an array of solid, liquid and gaseous products (Yaman 2004). Pyrolysis is an attractive option for managing solid waste because it results in significant volume reduction and the production of value added

Table 1. Olive oil processing products and by-products (adapted from IPROLIVE Project, 2003)

\begin{tabular}{llll}
\hline $\begin{array}{l}\text { Processing } \\
\text { Method }\end{array}$ & Output & & \\
\cline { 2 - 4 } & Oil $\left(\mathrm{kg} / \mathrm{Mg}_{\text {olive }}\right)$ & $\begin{array}{l}\text { Solid Waste } \\
\text { wet } \\
\left(\mathrm{kg} / \mathrm{Mg}_{\text {olive }}\right)\end{array}$ & $\begin{array}{l}\text { Waste Water } \\
\left(\mathrm{l} / \mathrm{Mg}_{\text {olive }}\right)\end{array}$ \\
\hline 3-phase & 200 & $500-600$ & $1000-1200$ \\
2-phase & 200 & $800-950$ & $170-185$ \\
\hline
\end{tabular}

biochar, bio-oil and gas. In comparison to direct combustion of biomass, the products of pyrolysis have higher energy density and therefore more economical to transport. The proportions of the pyrolysis products depend on the method followed, feedstock, residence time, heating rate, final temperature, particle size as well as the catalytic agent used (if any). Pyrolysis methods can be broadly classified as slow or fast. Slow pyrolysis is traditionally used to produce charcoal (Yaman 2004). On the other hand, fast pyrolysis is employed to maximise bio-oil production (Yoder, Galinato et al. 2011). 
Many researchers studied the potential of pyrolysis for treating the olive husk. While some researchers focused on its potential for producing hydrogen gas (e.g., (Caglar and Demirbas 2002; Taralas and Kontominas 2006; Encinar, Gonzalez et al. 2008)), others focused on the production of bio-oil (e.g., (Putun, Uzun et al. 2005; Sensoz, Demiral et al. 2006; Demirbas 2009)). The production of activated carbon from olive husk has also been studied (e.g., (Khalil 1999; Bacaoui, Yaacoubi et al. 2001; El-Sheikh, Newman et al. 2004; Skodras, Diamantopouiou et al. 2007; Ioannidou, Zabaniotou et al. 2010)). The kinetics and emissions from the pyrolysis process of olive cake under different conditions were also studied (e.g., (Jauhiainen, Conesa et al. 2004; Chouchene, Jeguirim et al. 2010; Miranda, Roman et al. 2010)). However, the benefits of using the bio-char from the pyrolysis of olive husk as a carbon sink has often been oversighted.

This paper investigates some of the economic-environmental trade-offs in the production and end-use of the biochar and bio-oil from the olive husk in Australia. Special attention is paid to the impact of policies such as Renewable Energy Target, Carbon Tax and Carbon Sequestration Credits on agricultural lands.

\section{FORMULATION}

\subsection{Data Collection}

Data on different parameters of olive husk pyrolysis was collected from various sources in the literature and summarised in Appendix A (supplementary data). The yield and quality of the bio-oil depends, in part, on the final pyrolysis temperature. Fig 2 shows the yield of the three major products of pyrolysis versus final pyrolysis temperature. To estimate the yield of biochar and bio-oil, empirical yield functions were fitted to the data as summarised in table 2.

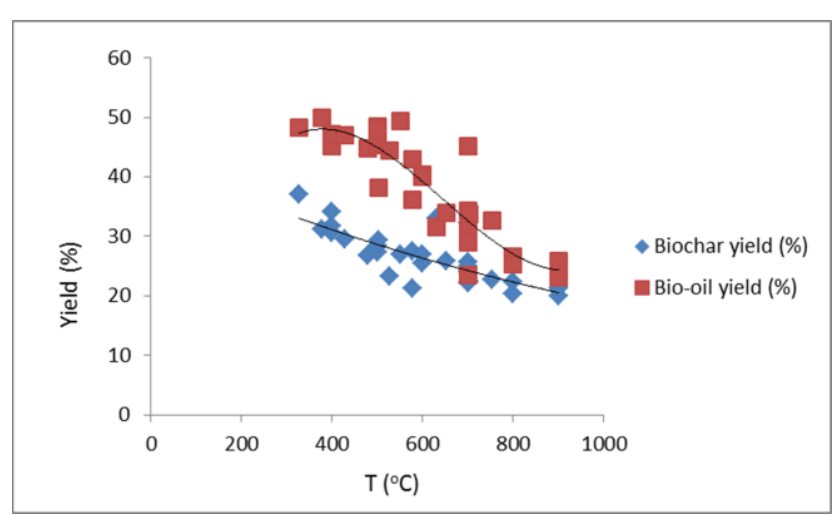

Figure 2. Yield of pyrolysis products at different temperatures.

Table 2. Empirical yield functions and goodness of fit

\begin{tabular}{lll}
\hline Descriptor & Biochar & Bio-oil \\
\hline Number of observations & 30 & 30 \\
Degrees of freedom & 29 & 29 \\
Fitted curve (yield) & $Y_{c}=43.348 e^{-0.0008 T}$ & $Y_{o}=3 \times 10^{-7} T^{3}-0.006 T^{2}+0.3242 T-4.9614$ \\
& $(1)$ & $(2)$ \\
$\mathrm{R}^{2}$ & 0.6102 & 0.788 \\
$\mathrm{X}^{2}$ & 8.6 & 17.9 \\
Significance level & $99.99 \%$ & $94.63 \%$ \\
\hline
\end{tabular}

\subsection{Products End Use}

In this paper it is assumed that bio-oil may be used as a substitute to heavy fuel. Two potential end uses of the biochar are considered: a) carbon sequestration through spreading of biochar on agricultural soils as a soil amendment and b) co-firing of biochar in a coal-fired power plant. Greenhouse gas emission offset through displacement of fossil fuel is used as environmental efficiency indicator. 


\subsection{Objectives Functions}

Two objectives need to be addressed; the environmental and the economic functions. As mentioned earlier, carbon offset is used as the environmental indicator. Therefore, the environmental objective function can be written as follows:

$\operatorname{Max} C_{\text {offset }}=a \times Y_{c} \times H H V_{c} \times \frac{E F_{\text {coal }}}{H H V_{\text {coal }}}+b \times Y_{c} \times C_{c} \times 3.667+Y_{o} \times H H V_{o} \times \frac{E F_{h f}}{H H V_{h f}}$

Where

$\mathrm{C}_{\text {offset}}$ : carbon emissions offset $\left(\mathrm{kg} \mathrm{CO}_{2 \text {-eq }}\right)$

a: fraction of the biochar used for energy production and soil amendment

$\mathrm{Y}_{\mathrm{c}}$ : biochar yield as in Eq 1, $(\mathrm{kg} / \mathrm{kg})$

$\mathrm{HHV}_{\mathrm{c}}$ : the higher heating value of the biochar $(\mathrm{MJ} / \mathrm{kg})$

$\mathrm{EF}_{\text {coal }}$ : the $\mathrm{CO}_{2}$ emission factor of coal $\left(\mathrm{kg} \mathrm{CO}_{2 \text {-eq }} / \mathrm{kg}\right.$ coal $)$

$\mathrm{HHV}_{\text {coal }}$ : the higher heating value of coal $(\mathrm{MJ} / \mathrm{kg})$

$\mathrm{b}$ : fraction of the biochar used as a soil amendment $(\mathrm{a}+\mathrm{b}=1)$

$\mathrm{C}_{\mathrm{c}}$ : is the fixed carbon content in biochar (\%)

$\mathrm{Y}_{\mathrm{o}}$ : Bio-oil yield as shown in Eq 2, $(\mathrm{kg} / \mathrm{kg})$

$\mathrm{HHV}_{\mathrm{o}}$ : the higher heating value of bio-oil $(\mathrm{MJ} / \mathrm{kg})$

$\mathrm{EF}_{\mathrm{hf}}$ : the $\mathrm{CO}_{2}$ emission factor of heavy oil $\left(\mathrm{kg} \mathrm{CO}_{2-\mathrm{eq}} / \mathrm{kg}\right.$ heavy oil)

$\mathrm{HHV}_{\mathrm{hf}}$ : the higher heating value of heavy oil $(\mathrm{MJ} / \mathrm{kg})$

The second objective is maximising the economic utility of the process which can be written as follows:

Max Profit $=\operatorname{Max}($ Rev - Cost $)$

$R e v=\left(a \times Y_{c} \times P_{c}+b \times Y_{c} \times P_{c S}+Y_{o} \times P_{o}\right)$

Where: $\mathrm{P}_{c}, \mathrm{P}_{c s}$ and $\mathrm{P}_{\mathrm{o}}$ are the price functions of biochar as coal substitute, biochar as soil amender and bio-oil as fuel oil substitute, respectively.

A generic price function may be formulated as follows:

$P=f\left(P_{e}, C_{p}, P_{m}, R E T_{c r}, D_{s u b}\right)$

$\mathrm{P}_{\mathrm{e}}$ : Energy content price based on substitution

$\mathrm{C}_{\mathrm{p}}$ : Carbon price mechanism

RET $_{\text {cr: }}$ Renewable energy target certificate price

$\mathrm{P}_{\mathrm{m}}$ : raw material price

$\mathrm{D}_{\text {sub }}$ : imperfect substitution adjustment

A generic cost function may also be formulated as follows:

Cost $=\left(C_{\text {cap }}+C_{m}+C_{e n v}+\sum C_{o p}\right)$

$\mathrm{C}_{\text {cap }}:$ is the capital cost

$\mathrm{Cm}$ : cost of raw material

$\mathrm{C}_{\mathrm{env}}$ : internalised environmental cost including the cost of disposal of residue and GHG emissions

$\sum C_{o p}$ : the sum of all other operational costs including labour, energy, maintenance, material drying, ..etc.

\subsection{Constraints}

To maximise the environmental benefits of the pyrolysis process, the amount of external fuel used should be minimised. Laird (2008) estimated the energy needed for the pyrolysis process to be around 15\% of the energy content of the biomass. Based on this, it can be estimated that around $3 \mathrm{MJ} / \mathrm{kg}$ of dried olive husk is required to drive the process. This energy can be derived from burning the pyrolysis gas produced. Zabanitou et al (2008) 
reported that the gas produced from the pyrolysis of olive husk has a HHV of around $11.9 \mathrm{MJ} / \mathrm{m}^{3}$ (Zabaniotou, Ioannidou et al. 2008). Keeping this in mind, it can be estimated that for the process to be self-fuelling, it has to run at temperatures of above $350{ }^{\circ} \mathrm{C}$. In the case of using biochar for sequestration on agricultural soils, it should be stable and needs to have sufficiently high porosity to be effective in retaining the nutrients from fertilisers. Lehmann noted that biochar produced at temperatures below $400{ }^{\circ} \mathrm{C}$ may not be suitable for improving soil fertility (Lehmann 2007). The following constraints therefore apply to both objective functions:

$400 \leq T \leq 700$

$Y_{c}, Y_{o} \geq 0$

$P_{c}, P_{o}, P_{c s} \geq 0$

The last constraint applies only to the economic objective function.

\section{POLICY IMPLICATIONS}

\subsection{Renewable Energy Target Scheme}

The Australian Renewable Energy Target scheme (RET) is designed to increase the share of electricity from renewable sources. The RET issues Renewable Energy Certificates (REC) to generators of renewable energy. These certificates can be sold in the market to electricity suppliers who use them to offset their 'green electricity' share. RET defines a range of renewable energy sources which are eligible to generate RECs. Such sources include biomass and other bio-sources such as biochar and bio-oil. Therefore, the use of the pyrolysis products in electricity generation may have the added economic incentive of generating RECs.

The producer of the biochar and bio-oil does not directly benefit from the RET because the electricity generator is entitled to the RECs. However, the scheme may create a market for the products which otherwise may not exist. Furthermore, as electricity retailers compete to fulfil their renewable energy commitments, the RET provides a market driver which may contribute positively to aid the adoption as well as improve the prices of the products. As a rule, an REC is generated for every $\mathrm{MW}_{\mathrm{e}}$ produced. However, the actual environmental benefit depends on the fuel displaced. For example, in Victoria where $48 \%$ of the olive husk is produced, the grid mix is heavily dependent on brown coal. As such, the displacement of $1 \mathrm{MW}_{\mathrm{e}}$ in Victoria reaps higher environmental benefit than in NSW where the grid mix is dominated by black coal. As a result, it creates discrepancy in the internalised price of the emissions.

\subsection{Carbon Tax (CT)}

Placing a price on carbon has become an eminent reality. The Australian government is pushing hard with its policy of carbon tax as a mechanism to charge a price on $\mathrm{CO}_{2}$ emissions. It has recently announced a price of $\$ 23$ per $\mathrm{Mg}$ of $\mathrm{CO}_{2}$ emitted. The tax, if passed in its current form, will only apply to the biggest emitters which include electricity generators but exclude petrol for passenger cars, small and medium enterprises and light transport vehicles. As a result, the tax in its current format will add another driver which favours 'electricity generation' from renewable resources such as pyrolysis products. By displacing the fossil fuel with bio-oil or biochar, electricity generators will avoid paying the tax for the proportion that is displaced. This might as well result in achieving the RET target as discussed earlier. The question, therefore, is how will the policy deal with the potential double counting resulting from the generation of RECs and avoided carbon tax? Another question that may be raised is how does the carbon tax price correlate to the internalised $\mathrm{CO}_{2}$ emissions price resulting from the RET scheme? Although, it is difficult to answer these questions at the moment until the full details of the tax are made public. It is clear that the existence of carbon price of one form or another will contribute positively to the adoption and enhance the prices of renewable energy sources such as bio-oil.

\subsection{Carbon Sequestration Credits}

Many researchers discussed the advantages of using biochar as a soil amendment. One advantage that is particularly important in this discussion is the long term sequestration of carbon in agricultural soils. The Kyoto Protocol defines two mechanisms for $\mathrm{CO}_{2}$ reduction: the first is through 'Policies and Measures' and the second is through 'Sinks Enhancements' (Lehmann, Gaunt et al. 2006). Nevertheless, current government policies do not provide a mechanism to credit farmers for such activities. As a result this alternative may be disadvantaged. Based on Eq 6, the achievable price of the biochar for this purpose is limited to the raw material price which the farmer is willing to pay for it as a soil amendment.

With the lack of financial incentives, the adoption of this alternative despite its environmental benefits may be limited. However, the new Carbon Tax mechanism is planned to eventually give way to an emissions trading scheme (ETS) by mid-2015. Under the planned ETS, emitters are allowed to offset their emissions by purchasing permits in an international market. These credits include permits generated under the Clean 
Development Mechanisms (CDM) scheme. Therefore, an opportunity exists if the government were to recognise carbon sequestration on agricultural soils as CDM under the 'Sinks Enhancement' mechanism of the Kyoto Protocol. This may create the financial incentive to encourage farmers to adopt this alternative.

\subsection{Policy Consequences}

As obvious from Eqs $3 \& 5$, the decision on how much bio-oil or biochar should be produced is influenced on one hand by how much carbon offset can be achieved. On the other hand, existence of incentives through the RET and CT contribute positively to improving the potential achievable prices of biochar and bio-oil as energy products.

The model suggested in this paper comprises of two objective functions, environmental and economic. If the economic objective is taken as the leading objective while the environmental objective is made as the following objective, the outcomes may be severely skewed towards energy use of the end products. The effect of the policy can be reduced by making the environmental function as a leading objective. However, in this case, the adoption of the technology by farmers may be compromised if the process fails to deliver tangible economic benefits to them.

\section{CONCLUSION}

The trade-offs between the production of bio-oil and biochar as well as the end use of the pyrolysis products from the olive husk are discussed based on a conceptual environmental-economic optimization model. The carbon pricing mechanism is an important component of the price function which is central to determining the economic potential of the pyrolysis process. This, in turn, plays an important role in determining the outcome of the environmental objective. The current government policies indirectly favour energy utilisation of pyrolysis products. However, the production of bio-char as a soil amendment may benefit greatly if the emission trading scheme (ETS) resulting from the new Carbon Tax were to include carbon sequestration as a 'Sink Enhancement' mechanism.

\section{REFRENCES}

Arjona, R., A. Garcia, et al. (1999). "The drying of alpeorujo, a waste product of the olive oil mill industry." Journal of Food Engineering 41(3-4): 229-234.

Bacaoui, A., A. Yaacoubi, et al. (2001). "Optimization of conditions for the preparation of activated carbons from olive-waste cakes." Carbon 39(3): 425-432.

Caglar, A. and A. Demirbas (2002). "Hydrogen rich gas mixture from olive husk via pyrolysis." Energy Conversion and Management 43(1): 109-117.

Chouchene, A., M. Jeguirim, et al. (2010). "Study on the emission mechanism during devolatilization/char oxidation and direct oxidation of olive solid waste in a fixed bed reactor." Journal of Analytical and Applied Pyrolysis 87(1): 168-174.

Dally, B. and P. Mullinger (2002). Utilization of Olive Husks for Energy Generation: A Feasibility Study, University of Adelaide.

Demirbas, A. (2009). "Fuel Properties of Pyrolysis Oils from Biomass." Energy Sources Part a-Recovery Utilization and Environmental Effects 31(5): 412-419.

El-Sheikh, A. H., A. P. Newman, et al. (2004). "Characterization of activated carbon prepared from a single cultivar of Jordanian Olive stones by chemical and physicochemical techniques." Journal of Analytical and Applied Pyrolysis 71(1): 151-164.

Encinar, J. M., J. F. Gonzalez, et al. (2008). "Two stages catalytic pyrolysis of olive oil waste." Fuel Processing Technology 89(12): 1448-1455.

Ioannidou, O. A., A. A. Zabaniotou, et al. (2010). "Preparation of activated carbons from agricultural residues for pesticide adsorption." Chemosphere 80(11): 1328-1336.

Jauhiainen, J., J. A. Conesa, et al. (2004). "Kinetics of the pyrolysis and combustion of olive oil solid waste." Journal of Analytical and Applied Pyrolysis 72(1): 9-15.

Kavdir, Y. and D. Killi (2008). "Influence of olive oil solid waste applications on soil pH, electrical conductivity, soil nitrogen transformations, carbon content and aggregate stability." Bioresource Technology 99(7): 2326-2332.

Khalil, L. B. (1999). "Porosity characteristics of chars derived from different lignocellulosic materials." Adsorption Science \& Technology 17(9): 729-739.

Lehmann, J. (2007). "Bio-energy in the black." Frontiers in Ecology and the Environment 5(7): 381-387.

Lehmann, J., J. Gaunt, et al. (2006). "Bio-char sequestration in terrestrial ecosystems - A review." Mitigation and Adaptation Strategies for Global Change 11: 403-427. 
Miranda, T., S. Roman, et al. (2010). "Emissions from thermal degradation of pellets with different contents of olive waste and forest residues." Fuel Processing Technology 91(11): 1459-1463.

Nair, N. G. and J. Markham (2008). Recycling Solid Waste from the Olive Oil Extraction Process. Kingston, ACT, Australia, RIRDC.

Putun, A. E., B. B. Uzun, et al. (2005). "Bio-oil from olive oil industry wastes: Pyrolysis of olive residue under different conditions." Fuel Processing Technology 87(1): 25-32.

Roig, A., M. L. Cayuela, et al. (2006). "An overview on olive mill wastes and their valorisation methods." Waste Management 26(9): 960-969.

Sensoz, S., I. Demiral, et al. (2006). "Olive bagasse (Olea europea L.) pyrolysis." Bioresource Technology 97(3): 429-436.

Skodras, G., I. Diamantopouiou, et al. (2007). "Enhanced mercury adsorption in activated carbons from biomass materials and waste tires." Fuel Processing Technology 88(8): 749-758.

Taralas, G. and M. G. Kontominas (2006). "Pyrolysis of solid residues commencing from the olive oil food industry for potential hydrogen production." Journal of Analytical and Applied Pyrolysis 76(1-2): 109116.

Yaman, S. (2004). "Pyrolysis of biomass to produce fuels and chemical feedstocks." Energy Conversion and Management 45(5): 651-671.

Yoder, J., S. Galinato, et al. (2011). "Economic tradeoff between biochar and bio-oil production via pyrolysis." Biomass and Bioenergy 35(5): 1851-1862.

Zabaniotou, A., O. Ioannidou, et al. (2008). "Experimental study of pyrolysis for potential energy, hydrogen and carbon material production from lignocellulosic biomass." International Journal of Hydrogen Energy 33(10): 2433-2444. 